\title{
Evidência de Validade da Medida de Atenção Plena pela Relação com Outras Variáveis
}

\author{
Jeferson Gervasio Pires ${ }^{1}$ \\ Carlos Henrique Sancineto da Silva Nunes' \\ Maiana Farias Oliveira Nunes ${ }^{1}$ \\ Marcelo Marcos Piva Demarzo ${ }^{2}$ \\ Maria Luiza Bianchi ${ }^{\top}$ \\ Mônica Monteiro Kotzias' \\ Giovania Mitie Maesima Cunha \\ ${ }^{1}$ Universidade Federal de Santa Catarina (UFSC), Florianópolis, SC \\ ${ }^{2}$ Escola Paulista de Medicina da Universidade Federal de São Paulo, São Paulo, SP
}

\begin{abstract}
Resumo
Atenção plena é uma qualidade da consciência que surge ao prestar atenção às experiências percebidas no momento presente. Este artigo objetiva buscar evidências de validade da Medida de Atenção Plena (MAP) pela sua relação com outros construtos. Participaram 553 brasileiros adultos, a maioria universitários e com média de idades de 23,15 anos (DP=7,34), os quais responderam às MAP, Bateria Fatorial de Personalidade, Escala de Afetos Positivos e Afetos Negativos, Escala de Satisfação de Vida, Bateria Psicológica da Atenção e à Medida de Inteligência Emocional. Foram efetuadas correlações entre os escores da MAP e os demais instrumentos. Encontraram-se correlações significativas entre mindfulness e os traços abertura e realização, afeto negativo e satisfação de vida. Atenção alternada, concentrada e geral e inteligência emocional mostraram-se associadas ao fator mindfulness. Conclui-se que a MAP possui evidências favoráveis de validade pela relação com outras variáveis, entretanto, novos estudos são demandados com o instrumento.

Palavras-chave: testes psicológicos, vigilância, psicologia positiva
\end{abstract}

\begin{abstract}
Validity Evidence of a Measurement of Mindfulness, Based on its Relations to Other Variables
Abstract

Mindfulness is a quality of consciousness, which may occur when one is intentionally aware of inner experiences. In this study, we have gathered validity evidence for a Measurement of Mindfulness (MAP), based on its relation to other variables $(\mathrm{N}=553)$. Participants included 553 Brazilian adults, mostly undergraduate students, with a mean age of 23.15 years (SD 7.34). We correlated the z-scores of MAP with the scores of the Factorial Battery of Personality, Positive affect and Negative Affect Scales, Satisfaction with Life Scale, Psychological Battery of Attention, and Measurement of Emotional Intelligence. Correlations were made between the MAP and other instruments. Significant correlations were found between mindfulness and the traits Openness and Satisfaction, negative affect and life satisfaction. Focused, alternating and general attention showed significant associations with mindfulness. Furthermore, we observed significant associations of emotional intelligence with mindfulness and novelty seeking. We conclude that the MAP is a valid instrument based on its relations to other variables; however, further studies with this instrument are necessary.
\end{abstract}

Keywords: Psychological tests; vigilance; positive psychology

\section{Evidencia de Validez de la Medida de Atención Plena por la Relación con Otras Variables}

Resumen

Atención Plena es una cualidad de la conciencia que surge al prestar atención a las experiencias internas percibidas en el presente momento. Este artículo tiene por objetivo buscar evidencias de validez de la Medida de Atención Plena en su relación con otros constructos. Participaron 553 brasileños adultos, mayoría universitarios y con edad media de 23,75 años; DP=7,34), los cuales respondieron Medida de Atención Plena (MAP), Batería Factorial de Personalidad, Escala de Afectos Positivos y Afectos Negativos, Escala de Satisfacción de vida, Batería Psicológica de Atención, y Medida de Inteligencia Emocional. Fueron efectuadas correlaciones entre los resultados de MAP y los demás instrumentos. Se encontraron correlaciones significativas entre Atención Plena y los trazos de Apertura y Realización, afecto negativo y satisfacción de vida. Atención alternada, concentrada y general, e inteligencia emocional se mostraron asociadas al factor Atención Plena. El estudio permite concluir que MAP posee evidencias favorables de validez por su relación con otras variables, mientras tanto, nuevos estudios son necesarios con el instrumento. Palabras-clave: tests psicológicos; vigilancia; psicología positiva

\section{Introdução}

O estado de atenção plena (mindfulness) pode ser definido como uma qualidade da consciência que surge ao prestar atenção, de forma intencional e com postura de não julgamento, às experiências (pensamentos, sensações e sentimentos) que surgem no momento presente (Kabat-Zinn, 2003). Para Brown e 
Ryan (2003), a atenção plena é um atributo da consciência, capaz de promover bem-estar psicológico e que envolve awareness e atenção. Awareness diz respeito ao monitoramento das experiências internas (sensações, sentimentos, pensamentos) e do ambiente, o que também inclui seu aspecto não elaborado (como perceber-se pensando), ao passo que a atenção envolve, exclusivamente, a "seleção" da experiência (Baars, 1997), promovendo aumento de sensibilidade a estímulos delimitados (Brown \& Ryan, 2003).

Langer (2014), por sua vez, refere-se à atenção plena como um estado não automático de funcionamento cognitivo e comportamental, o que seria diferente de ser guiado por um modo automático (condição padrão), necessitando ser, por isso, intencionalmente "ativado" durante as atividades cotidianas. Para essa autora, o fenômeno é explicado a partir dos estados mindful e mindless. O mindful é como um estado de pleno engajamento com o momento presente, com o ambiente e com a atividade que se realiza, condição que leva à maior sensibilidade e se opõe ao estado mindless, sendo esse um estado de "modo de funcionamento automático".

Diferentes compreensões têm disso empregadas ao fenômeno mindfulness (Siegling \& Petrides, 2014), podendo variar de um traço a um estado. Enquanto um traço refere-se a uma característica individual, que apresenta variação entre as pessoas, e que se mantém com alguma estabilidade ao longo do tempo, para ser considerado um estado, o fenômeno deve estar associado a um contexto, podendo não representar estabilidade temporal, mas sim uma ocorrência. Alguns autores apontam a necessidade de melhor compreender as diferenças entre o estado e o traço mindfulness (Bergomi, Tschacher, \& Kupper, 2012), até porque as diferentes compreensões para o fenômeno afetam a sua operacionalização nos instrumentos de medida (Pires, 2016).

Estudos envolvendo análises fatoriais de itens desenvolvidos para avaliar o fenômeno mindfulness (seja como uma característica humana ou um estado de funcionamento) tem reportado extrações unifatoriais, bifatoriais, com quatro fatores, e também com cinco componentes (ver Pires, 2016). Além da variedade de estruturas, ocorre de mesmos componentes receberem diferentes nomenclaturas, causando a realidade de jingle-jangle fallacies, a exemplos de "agir com consciência" e "atenção"; ou "aceitar as experiências", "aceitar sem julgar" e "não reagir às experiências". Entretanto, deve-se destacar que a estrutura composta por um fator geral mindfulness, associado a outros componentes cognitivos, tem sido a mais respaldada cientificamente, nas diferentes culturas e populações (Baer et al., 2006).

Apesar disso, pesquisas que avaliam os efeitos de práticas de mindfulness, tanto com grupos clínicos quanto não clínicos, têm destacado o papel desse fenômeno em demandas que envolvem regulação das emoções, sendo descritos impactos positivos na saúde e qualidade de vida daqueles que participam. Estudos registraram, por exemplo, que os escores em atenção plena possuem associação com os traços de personalidade, o bem-estar subjetivo e a inteligência emocional (Brown \& Ryan, 2003; Giluk, 2009), fenômenos que serão destacados na sequência.

Em relação aos traços de personalidade, estes se referem a padrões cognitivos, comportamentais e emocionais, que se mantêm com alguma estabilidade ao longo do tempo nos indivíduos. Há variados modelos que buscam organizar hierarquicamente os traços de personalidade, mas, gradualmente, houve uma crescente adoção do modelo dos Cinco Grandes Fatores (John, Naumann, \& Soto, 2008). Nesse modelo, os traços são organizados e descritos por cinco domínios amplos, abertura, realização, extroversão, socialização e neuroticismo.

Abertura se associa com o comportamento exploratório e a obtenção de novas experiências (Nunes et al., 2010). Indivíduos com altos escores em Abertura tendem a ser curiosos, imaginativos e possuem valores não convencionais. Realização ou "Conscienciosidade" indica a capacidade de organização, de produção e de cumprimento de metas das pessoas, estando, por isso, associado a fatores como persistência e motivação. Indivíduos altos em Realização são organizados, persistentes, confiáveis e concluem o que iniciam. Socialização ou Amabilidade diz respeito à profundidade e à qualidade das relações interpessoais, de forma que pessoas altas nessa dimensão são mais generosas, bondosas, tendem a confiar nos demais e raramente suspeitam das intenções alheias. Extroversão refere-se à quantidade e intensidade das relações interpessoais, sugerindo o quanto o sujeito é comunicativo, falante e assertivo. Pessoas altas em Extroversão são sociáveis, falantes e dominantes. Por fim, neuroticismo indica o nível de ajustamento emocional das pessoas. Altos escores sugerem maior propensão a experimentar sofrimento emocional, ideias e emoções negativas, de preocupação com a opinião dos demais e ansiedade.

Uma vez que a personalidade tende a modular cognições, emoções e comportamentos, entende-se que esse fenômeno influencia a atenção plena. Em linha 
com isso, Khazan (2013), em seu manual sobre a utilização de práticas da meditação mindfulness e biofeedback no contexto clínico, enfatizou a importância de conhecer características da personalidade dos clientes, as quais podem prejudicar ou contribuir com a motivação e/ou desistência da terapia. Associado a isso, Teper e Inzlicht (2013) demonstraram que indivíduos com escores mais elevados no traço mindfulness são menos sujeitos às recompensas imediatas, expressão típica das dimensões humanas extroversão e realização.

Seguindo a lógica, Shapiro (2009) leciona que a intencionalidade é um requisito para o estado mindfulness, de forma que, para atingi-lo, deve-se treiná-lo diariamente, de maneira intencional. Isso traz destaque para a persistência, característica típica do traço Realização. Colaborando com o argumento, Van den Hurk et al. (2011) concluíram que a prática de meditação mindfulness está associada com níveis mais elevados de curiosidade e de receptividade para novas experiências. Para esses autores, os traços de personalidade possuem certo papel moderador na motivação e mesmo manutenção da prática meditativa, o que foi corroborado por Vibe et al. (2015).

No tocante às associações entre o fenômeno mindfulness e personalidade, na construção da Escala de Atenção e Consciência Mindfulness (MAAS), Brown e Ryan (2003) reportaram correlações fracas, porém significativas $(r=0,18$, NEO-PI) e $(r=0,19$, NEO-FFI) com Abertura, além de correlações negativas e moderadas $(r=-0,56$, NEO-PI) e $(r=-0,33$, NEO-FFI) com neuroticismo. No estudo de construção do Questionário das Cinco Facetas de Mindfulness (FFMQ), Baer et al. (2006) reportaram correlações com neuroticismo variando entre fraca ( $r=-0,23$, faceta observar) e moderada $(r=-0,44$, faceta agir com consciência), porém, negativas e significativas. Abertura apresentou correlações entre fraca e negativa ( $r=-0,07$, faceta não julgar) e moderada positiva ( $r=0,42$, faceta observar).

Igualmente, Giluk (2009) demonstrou, em sua meta-análise, correlações significativas entre mindfulness, traços de personalidade e afetos. Sobre personalidade, neuroticismo apresentou a maior correlação negativa com mindfulness $(r=-0,58)$. Extroversão apresentou a menor correlação positiva $(r=$ $0,15)$ e o fator Realização apresentou a correlação com a mais elevada magnitude positiva com atenção plena $(r=0,44)$. Socialização obteve correlação fraca $(r=$ $0,30)$, resultado similar ao obtido com abertura $(r=$ $0,20)$. Vale mencionar que, de forma geral, esse padrão de associação pode ser resgatado em outros estudos
(Hollis-Walke \& Colosimo, 2011; Latzman \& Masuda, 2013; Siegling \& Petrides, 2014).

Outro construto que tem sido relacionado com mindfulness é o bem-estar subjetivo (BES) (Brown \& Ryan, 2003; Giluk, 2009). Diener, Lucas e Oishi (2005) referem-se ao BES como a avaliação cognitiva e afetiva que uma pessoa faz em relação à própria vida. No aspecto cognitivo, está a satisfação de vida (Hutz, Zanon, \& Bardagi, 2014) e, no emocional, está o balanço entre os afetos positivos e negativos (Zanon \& Hutz, 2014). Os afetos dizem respeito à intensidade e frequência com que se vivenciam as emoções positivas e negativas (Zanon, Dellazzana-Zanon, \& Hutz, 2014). Para esses autores, pessoas que sentem repetidamente emoções negativas (tristeza, irritação, preocupação) autorrelatam mais afetos negativos, e aqueles que experimentam mais emoções positivas indicam níveis mais elevados de afetos positivos. Porém, a satisfação de vida descreve a avaliação subjetiva do quão satisfeito se está com a própria vida de forma geral e envolve diversos domínios como o trabalho e saúde (Hutz, Zanon, $\&$ Bardagi, 2014).

Entende-se que indivíduos com altos escores em atenção plena apresentam maior flexibilidade em ambas as avaliações do BES, indicando maior satisfação geral com a vida, além de equilíbrio na percepção de afetos positivos e negativos. A esse respeito, pode-se pensar que a postura de maior consciência, curiosidade e aceitação das próprias experiências, associada à compreensão de que estas (positivas ou negativas) são transitórias (Lau et al., 2006), pode contribuir para que as emoções negativas de um domínio da vida não interfiram nos demais.

No tocante às evidências empíricas de associação entre o estado mindfulness e construtos da Psicologia Positiva, Brown e Ryan (2003), por exemplo, reportaram correlações fracas, mas estatisticamente significativas, com afeto positivo $(r=0,30)$ e $(0,39)$, e fracas a moderadas com afeto negativo $(r=-0,39$ e $-0,43)$, em duas amostras de adultos. É válido mencionar que essas magnitudes negativas e positivas denotam o equilíbrio entre os afetos. Satisfação com a vida também resultou em correlação fraca, variando entre 0,26 e 0,30. Esses padrões de associação foram corroborados em outros estudos internacionais, inclusive com magnitudes ainda mais fracas (Giluk, 2009; Jimenez, Niles, \& Park, 2010; Kiken \& Shook, 2014) e também brasileiros (Barros et al., 2014; 2015), indicando que os fatores "Observar as experiências", "Não reagir às experiências" e "Agir com consciência", são os aspectos do construto mindfulness 
mais relacionados ao componente cognitivo de BES. Além disso, destaca-se que tanto esses aspectos quanto o escore total em mindfulness são mais proeminentes em indivíduos com níveis elevados de afeto positivo.

Esse conjunto de resultados indica que o estado mindfulness e o BES são variáveis independentes umas das outras, apesar de que ter altos escores em mindfulness parece contribuir com uma visão mais abrangente dos diversos domínios da vida, o que poderia gerar certa percepção de equilíbrio entre as diversas experiências, positivas e negativas, vivenciadas cotidianamente. Dessa forma, pode-se dizer que o estado mindfulness, em alguma medida, atua no nível da regulação das emoções.

Pesquisas também têm sugerido associações entre mindfulness e inteligência emocional (Brown \& Ryan, 2003, Baer et al., 2006). Salovey e Mayer (1995) descrevem as emoções como respostas organizadas e que envolvem múltiplos subsistemas (cognitivo, fisiológico, emocional). Leahy et al. (2013), além de sugerirem que as emoções têm a função de revelar as necessidades básicas das pessoas, acrescentam que a consciência e a aceitação das emoções, componentes do estado mindfulness, são habilidades da regulação emocional (Leahy et al., 2013). Para Brown e Ryan (2003), os estudos sobre autorregulação buscam entender o papel da consciência e da atenção no processamento e manutenção do repertório de funcionamento psicológico e comportamental, de forma que, para esses autores, a consciência seria um facilitador do reconhecimento e da escolha de comportamentos mais consistentes com as necessidades e interesses individuais.

Em 1999, Siqueira, Barbosa e Alves operacionalizaram a inteligência emocional, considerando a teoria de Daniel Goleman, em cinco componentes: autoconsciência, automotivação, autocontrole, empatia e sociabilidade. Autoconsciência, que diz respeito à facilidade em identificar, nomear, lidar e avaliar os próprios sentimentos, tem sido referida como o componente da inteligência emocional mais associado ao estado Mindfulness (Brown \& Ryan, 2003; Baer et al., 2006). No estudo de Brown e Ryan (2003), por exemplo, foi relatada correlação positiva moderada e significativa entre o instrumento MAAS e o fator autoconsciência $(r=0,45)$. No estudo de validade incremental da MAAS, as correlações obtidas com o mesmo fator foram fracas, porém significativas $(r=$ $0,34)$ e $(r=0,37)$. Esse padrão de associação foi corroborado no estudo de Baer et al. (2006).

Desses estudos, pode-se concluir que a autoconsciência é um componente fundamental do estado mindfulness. Entretanto, deve-se lembrar que há pesquisas indicando que um componente da autoconsciência é pouco adaptativo e se refere à ruminação das experiências (Boyraz \& Waits, 2015; DaSilveira, DeSouza, \& Gomes, 2015). Associado a isso, está a aceitação das próprias experiências, ou autoaceitação, que também é um fator do estado mindfulness. A seu respeito, no estudo de validação de um questionário de aceitação (AAQ-II) para a cultura espanhola ( $N=132)$, Ruiz et al. (2013) encontraram correlações significativas e negativas com todos os quatro componentes de mindfulness do instrumento KIMS (Baer et al., 2004). A associação com a faceta aceitação foi negativa, moderada e significativa $(r=-0,49)$. Colaborando com a discussão, no estudo de Boyraz e Waits (2015), a autoaceitação resultou em correlação fraca $(r=-0,16)$, porém, negativa e significativa com empatia. As autoras entenderam que a ruminação das experiências possui algum papel na empatia, de forma que, segundo elas, é possível que os mais suscetíveis à ruminação percebam com mais facilidade situações estressantes de outras pessoas, imaginando sua reação a situações similares vividas no passado, aumentando dessa forma, a empatia.

Nessa mesma direção, de carência de pesquisas, pouco tem sido produzido, cientificamente, para investigar associações entre mindfulness e atenção. A atenção, em seu modelo tradicional, tem sido definida como a capacidade de se organizar e processar ativamente informações disponíveis nos sentidos (Sternberg, 2008). Rueda e Monteiro (2013), inclusive, indicam que o construto tem sido operacionalizado de formas distintas, apesar de tal variação ser de ordem taxonômica. Esses autores propõem um modelo composto por três habilidades, quais sejam: concentrar, dividir e alternar. Para Rueda (2013) a atenção concentrada refere-se à capacidade de focar-se em uma única tarefa ou objeto, eliminando estímulos distratores. A atenção dividida, por sua vez, refere-se ao efetuar duas tarefas, ou manter-se concentrado em dois ou mais estímulos, ao mesmo tempo. A atenção alternada é a habilidade de alternar o foco da atenção e a concentração, ora para uma atividade ou objeto, ora para outro.

Tendo em vista que essas habilidades da atenção são importantes em diferentes momentos da vida, pode-se hipotetizar que a consciência sobre elas poderia promover a sua regulação (Bishop et al., 2004). Enquanto por um lado a consciência poderia recrutar a atenção concentrada e dessa forma, refletir o engajamento com a atividade presente, focando em uma experiência por vez, tal como preconizado por Baer et 
al. (2004); por outro lado, a vagueação da atenção poderia ser considerada um atributo contrário à mindfulness (Mrazek et al., 2012) ou da consciência sobre a atividade da atenção. Nessa condição, dividir a atenção, por exemplo, poderia ser um indicativo de vagueação, de forma que ao utilizar-se mais dessa habilidade, menos se engaja com a atividade corrente. Assim, entende-se que sujeitos com maiores escores em mindfulness também possuem maior habilidade de regular a atenção, mostrando-se hábeis em focar, dividir ou alterná-la, quando necessário.

Apesar da carência de pesquisas envolvendo o fenômeno mindfulness e as habilidades típicas da atenção, na pesquisa de Schmertz (2006), dividir, alternar e sustentar o foco da atenção mostraram-se independentes dos escores em mindfulness, considerando-se três escalas (CAMS-R, KIMS e MAAS). Apenas a quantidade de erros e omissões mostraram-se inversamente proporcionais ao escores totais em mindfulness, com exceção da faceta "agir com consciência". Entretanto, devemos lembrar que esses instrumentos avaliam mindfulness como um traço, e possivelmente, instrumentos que avaliem o construto como um estado poderão apresentar associação mais forte entre os construtos, já que as habilidades da atenção podem ser aumentadas "durante o estado", e não de "forma geral" na vida (Schmertz, 2006).

Conforme foi mapeado, o estado mindfulness e seus componentes apresentam relações documentada com construtos como personalidade, bem-estar subjetivo, atenção, e inteligência emocional. Além disso, identifica-se a relevância desse construto em contextos variados, que englobam o trabalho, a educação, a saúde e a qualidade de vida. Nesse sentido, de acordo com os standards mencionados pela International Test Comission (ITC, 2014), o uso de medidas com robusto conjunto de evidências de validade, contextualizadas e adequadas para o uso com o público alvo desejado é essencial para a prática do profissional que atua nesses contextos. Isso inclui profissionais que trabalham com o fenômeno mindfulness.

Apesar das diferentes definições para o construto, uma série de questionários e medidas para o avaliarem tem sido desenvolvida internacionalmente, principalmente na língua inglesa, contribuindo tanto com as intervenções em saúde e educação baseados em mindfulness, quanto com a produção de maior conhecimento envolvendo o fenômeno (Pires, 2016). Além disso, os dados levantados com as escalas de mindfulness, tanto na prática profissional quanto em pesquisa em
Psicologia e outras áreas da saúde, podem contribuir com o desenvolvimento e mesmo avaliação de programas para a promoção e monitoramento do estado mindful nos diversos contextos. No entanto, apesar de algumas medidas de mindfulness já estarem validadas para o Português do Brasil (Pires, 2016), ainda existem poucos instrumentos desse construto que tenham sido desenvolvidos no contexto étnico e cultural brasileiro, preocupação que poderia beneficiar estudos de efetividade e de elucidação dos mecanismos envolvidos em populações nacionais.

Considerando que a Medida de Atenção Plena (MAP), desenvolvida para a população brasileira, possui evidência de validade de conteúdo, de validade baseada em sua estrutura interna e de critério (Pires, 2016), estudos que possam verificar a associação entre a variável latente medida na MAP e outros construtos poderiam contribuir como fontes de validade ao instrumento. Cumpre dizer que na elaboração da MAP, o construto foi entendido de forma integrativa, tendo sido descrito como um estado que envolve a consciência e a atenção (Bishop et al., 2004), no qual se está intencionalmente: a) aberto para perceber as próprias experiências, sem atitude de pré-julgamento (Kabat-Zinn, 2003), além de b) produzir novidades no ambiente, tornando-se mais sensível ao mesmo e consciente por diferentes perspectivas (Langer, 2014).

As dimensões que a MAP avalia são mindfulness, Atenção e sua regulação, Aceitação e Produção de novidades. Mindfulness indica o quão consciente, aberto, curioso e sensível se está em relação às próprias experiências, às atividades e ao ambiente. Esse componente também diz respeito ao monitoramento das experiências, tanto de forma intencional, envolvendo atitudes como observar e descrever, quanto de maneira não elaborada, como dar-se conta, perceber-se ou pegar-se fazendo ou pensando em algo. Atenção refere-se a utilizar as diferentes habilidades de concentrar, alternar e dividir a atenção, voluntariamente, seja para produzir maior consciência ou para promover sua autorregulação. Aceitação é o domínio que indica o quanto as pessoas aceitam as próprias experiências e as deixam ser do jeito que são, sem evitá-las ou alterá-las. Produção de novidades refere-se ao ato de promover a consciência, a partir da exploração e descoberta de novos elementos no ambiente e no contexto. Essa atitude, também conhecida como "mente de principiante", contribui para que o sujeito não vagueie ou seja guiado por um modo automático de funcionamento. 
Levando-se em conta a necessidade de medidas adequadas para o contexto brasileiro, neste artigo objetiva-se buscar evidências de validade para a MAP, baseando-se em sua relação com outras variáveis, sendo elas: traços de personalidade, atenção, bem-estar subjetivo e inteligência emocional. Ademais, algumas hipóteses podem ser feitas no âmbito das associações, em observância às pesquisas apresentadas.

A respeito das correlações com traços de personalidade, são esperadas correlações significativas e negativas entre mindfulness e neuroticismo, além de positivas com abertura, realização e extroversão, variando entre fraca e moderada, e correlações fracas com socialização. Sobre o bem-estar subjetivo, espera-se obter correlações positivas e significativas com afeto positivo e satisfação de vida, além de negativa e pelo menos moderada com afeto negativo. Espera-se obter correlações positivas, fracas e moderadas, com quatro domínios da inteligência emocional (empatia, automotivação, autocontrole e autoconsciência); além de correlação fraca com sociabilidade. A respeito das associações com atenção, hipotetiza-se obter correlações fracas com atenção geral, concentrada e alternada, ao passo que a atenção dividida deverá estar menos associada com o fator Mindfulness, já que indica funcionamento multitasking.

\section{Método}

\section{Participantes}

Participaram deste estudo 553 sujeitos adultos, estudantes universitários dos cursos de Psicologia ou Naturologia, os quais foram divididos em quatro grupos, com o objetivo de realizar a aplicação espiralada dos cinco instrumentos. O primeiro grupo compôs-se por 146 adultos, sendo a maioria solteiros $(n=120)$, do sexo feminino $(n=122)$ e com idades entre 17 e 57 anos $(M=23,15, D P=7,34)$. Cento e trinta e três participantes estavam cursando a primeira graduação, ao passo que outros seis já possuíam algum curso superior, e três já eram mestres. O grupo respondeu a MAP e a Bateria Fatorial de Personalidade.

O segundo grupo compôs-se por 150 adultos, sendo a maioria solteiro $(n=123)$, do sexo feminino $(n=111)$, com idades entre 18 e 57 anos $(M=24,55$; $D P=7,29)$. A maioria desses participantes cursava a primeira graduação $(n=135)$, ao passo que sete já possuíam algum curso superior. Outros seis já possuíam o grau de mestre. O grupo respondeu a MAP, a Positive
Affect and Negative Affect Scales (PANAS) e a Escala de Satisfação de Vida.

O terceiro grupo compôs-se por 119 adultos, sendo a maioria solteiro $(n=99)$, e do sexo feminino $(n$ =97), com idades entre 18 e 57 anos $(M=24,71 D P=$ $8,49)$. Cento e quatro participantes cursavam a primeira graduação, ao passo que outros sete possuíam algum curso superior, e outros seis indicaram possuir grau de mestre. A maioria era residente em Santa Catarina $(n=$ 94), e outros 28 em São Paulo. Esse grupo respondeu a MAP e a Bateria Psicológica para Avaliação da Atenção.

O quarto grupo compôs-se por 138 adultos, a maioria solteiro $(n=115)$, do sexo feminino $(n=109) \mathrm{e}$ com idades entre 17 e 63 anos $(M=23,46 D P=7,59)$. A maior parte desses participantes $(n=133)$ cursava a primeira graduação, ao passo que uma pessoa já possuía curso superior; mesma frequência para mestrado e doutorado. Esse grupo respondeu a MAP e a Medida de Inteligência Emocional.

\section{Considerações Éticas}

Esta pesquisa foi aprovada pelo comitê de ética em pesquisas com seres humanos de uma universidade de (Informação retirada pela revista) (CAAE: 43086815.4.0000.0121). Participaram da pesquisa aqueles que concordaram com o termo de consentimento livre e esclarecido.

\section{Instrumentos}

Questionário Social e Demográfico. Foi composto por questões elaboradas para controle e mapeamento de variáveis sócio demográficas, tais como: sexo, idade e escolaridade, além de informações complementares sobre a experiência prévia com meditação.

Medida de Atenção Plena (MAP) (Pires, 2016). Composta por 47 itens de autorrelato, com o objetivo de avaliar comportamentos, crenças e características de quatro domínios da atenção plena: Mindfulness $(\alpha=0,88)$, atenção e sua regulação $(\alpha=0,84)$, aceitação $(\alpha=0,78)$ e produção de novidades $(\alpha=0,62)$. O respondente deve ler os itens e julgar o quanto se identifica com eles, utilizando-se de uma escala de cinco pontos, de forma que 1 representa "a frase não tem nada a ver com você" e o 5 "a frase tem tudo a ver com você". Deve-se deixar o item sem julgamento, caso não entenda seu conteúdo ou não saiba responder a respeito.

Bateria Fatorial de Personalidade (BFP). Publicada em 2010 por Nunes, Hutz e Nunes, visa medir traços de personalidade, baseando-se no 
modelo dos Cinco Grandes Fatores (CGF). Compõe-se por 126 itens que avaliam a concordância em relação ao item, em uma escala de sete pontos, sendo 1 "absolutamente não me descreve" e 7 "a sentença me descreve bem". São avaliados os seguintes domínios: a) extroversão; b) socialização; c) neuroticismo; d) realização; e) abertura à experiência. A precisão dos fatores da BFP obteve coeficientes variando entre $(\alpha$ $=0,74$ e $\alpha=0,89)$. A aplicação e a correção seguiram as normas descritas no manual do instrumento (Nunes et al., 2010).

Positive Affect and Negative Affect Scales (PANAS). É uma escala de autorrelato composta por 20 itens que visam medir o aspecto afetivo do bemestar subjetivo (BES), sendo dez itens para o afeto positivo e outros dez para o afeto negativo. A medida foi reformulada por Watson, Clark e Tellegen (1988), obtendo $(\alpha=0,86)$ para afeto positivo e $(\alpha=0,84)$ para o negativo, tendo sido adaptada para o Brasil por Hutz e Giacomoni, em 1996, (não publicado) apresentando alfa de $(\alpha=0,88)$ para o afeto positivo e $(\alpha=0,86)$ para o negativo. Os respondentes devem apontar o quanto sentem as emoções descritas nos itens, a partir de uma escala com cinco pontos, sendo que 1 representa nem um pouco e 5 extremamente.

Escala de Satisfação de Vida (ESV). Mede o aspecto cognitivo do bem-estar subjetivo. Originalmente construída por Diener, Emmons, Larsen e Griffin, em $1985(\alpha=0,87)$, foi adaptada por pesquisadores do Laboratório de Mensuração da Universidade Federal do Rio Grande do Sul (Hutz et al., 2014), apresentando ótimo nível de precisão $(\boldsymbol{\alpha}=0,91)$. Compõe-se de cinco itens de autorrelato, e uma escala de concordância de sete pontos, de forma que o ponto 1 representa discordo plenamente e o 7 concordo plenamente.

Bateria Psicológica para Avaliação da Atenção (BPA). Construída por Rueda (2013) para avaliar a capacidade geral da atenção (AG) e de seus três subtipos: Atenção Concentrada (AC), Atenção Dividida (AD) e Atenção Alternada (AA). O tempo da aplicação é de dois minutos para $\mathrm{AC}$, quatro minutos para $\mathrm{AD}$ e menos de três minutos para AA. O aplicativo Hybrid Stopwatch foi utilizado para controlar o tempo. A BPA possui evidência de validade pela comparação com outros instrumentos que também avaliam a atenção, cujos coeficientes variaram entre $(r=0,34)$ e $(r=$ $0,59)$ (Rueda \& Monteiro, 2013). A precisão estimada apresentou coeficientes satisfatórios (entre $\alpha=0,68 \mathrm{e}$ $0,89)$. A aplicação e a correção seguiram as instruções do manual do instrumento (Rueda, 2013).
Medida de Inteligência Emocional (MIE). Desenvolvida por Siqueira, Barbosa e Alves, em 1999, visa a medida de cinco componentes da inteligência emocional, baseados na teoria de Daniel Goleman, quais sejam: Empatia $(\alpha=0,87)$, Sociabilidade $(\alpha=$ $0,82)$, Automotivação $(\alpha=0,82)$, Autocontrole $(\alpha=$ $0,84)$ e Autoconsciência $(\alpha=0,78)$. Deve-se responder os 59 itens, baseando-se na frequência com que são emitidos os comportamentos listados, em uma escala de quatro pontos, sendo que 1 indica nunca e 4 sempre. A aplicação e a correção seguiram as instruções de Siqueira et al. (1999).

\section{Procedimentos}

Após a aprovação da pesquisa pelo comitê de ética, foram feitos contatos com coordenadores de cursos de Psicologia de diferentes regiões de Santa Catarina. Igualmente, foram contatados coordenadores de cursos de Naturologia, sendo um na grande Florianópolis e outro em São Paulo. Por essa via, foram acessados 558 participantes, os quais responderam aos instrumentos no formato clássico, em lápis e papel, em grupos de aproximadamente 30 alunos, durante uma aula. $\mathrm{Na}$ ocasião da coleta, o pesquisador explicava brevemente a pesquisa, apresentava o TCLE e então conduzia a atividade. Os participantes responderam a MAP, seguida do segundo e terceiro instrumento. O tempo médio total das aplicações variou de 1 h15min a 2 horas.

\section{Análise dos Dados}

Considerando a normalidade das distribuições obtidas, foram efetuadas correlações paramétricas entre a média dos escores z referentes à MAP e os escores nos demais instrumentos mencionados. Para as análises, utilizou-se o software Stata $12 \AA$.

\section{Resultados}

Mindfulness mostrou-se amplamente associado com os traços de personalidade, o bem-estar subjetivo, a atenção e a inteligência emocional. As magnitudes obtidas nessas associações podem ser apreciadas na Tabela 1.

No tocante aos traços de personalidade, neuroticismo resultou em correlações significativas e negativas, entre fraca e moderada, com os fatores atenção, aceitação e produção de novidades; e não resultou em associação significativa com o fator mindfulness. Abertura à experiência mostrou-se significativamente associada à mindfulness e produção de novidades, 
Tabela 1

Correlações de Pearson entre os Fatores da MAP e dos Demais Instrumentos

\begin{tabular}{|c|c|c|c|c|c|}
\hline & Mindfulness & Atenção & Aceitação & $\begin{array}{c}\text { Produção } \\
\text { de novidades }\end{array}$ & $\begin{array}{c}\text { Atenção } \\
\text { plena geral }\end{array}$ \\
\hline Neuroticismo & $-0,12$ & $-0,65^{* *}$ & $-0,42 * *$ & $-0,26 * * *$ & $-0,62^{* *}$ \\
\hline Abertura & $0,39 * *$ & 0,03 & $-0,07$ & $0,32^{* *}$ & $0,24 * * *$ \\
\hline Realização & $0,31 * *$ & $0,19 *$ & $-0,11$ & $0,24 * * *$ & $0,24 * * *$ \\
\hline Socialização & 0,09 & $0,23 * * *$ & 0,01 & 0,17 & $0,21 *$ \\
\hline Extroversão & 0,15 & 0,10 & 0,08 & $0,22^{* * *}$ & $0,21 * * *$ \\
\hline Afeto positivo & $0,16^{*}$ & 0,03 & $-0,14$ & 0,04 & 0,02 \\
\hline Afeto negativo & $-0,12$ & $-0,13$ & $-0,18^{*}$ & $-0,12$ & $-0,23^{* *}$ \\
\hline Satisfação com a vida & $0,23 * *$ & $0,27 * *$ & 0,11 & $0,23 * *$ & $0,34 * * *$ \\
\hline Atenção dividida & 0,16 & 0,11 & $-0,08$ & $0,20 *$ & 0,10 \\
\hline Atenção concentrada & $0,21 *$ & 0,12 & $-0,08$ & 0,01 & 0,09 \\
\hline Atenção alternada & $0,25^{* *}$ & 0,13 & & 0,10 & $0,18^{*}$ \\
\hline Atenção geral & $0,27 * *$ & $0,21 * *$ & $-0,02$ & 0,08 & $0,20 *$ \\
\hline Empatia & $0,49 * * *$ & $-0,02$ & $-0,40 * * *$ & $0,25^{* *}$ & 0,08 \\
\hline Sociabilidade & $0,21 *$ & 0,05 & $-0,08$ & $0,22^{* *}$ & $0,17^{*}$ \\
\hline Automotivação & $0,28^{* *}$ & 0,10 & $-0,15$ & $0,25^{* *}$ & $0,19 *$ \\
\hline Autocontrole & $0,23^{* *}$ & $0,21 *$ & & & $0,21 *$ \\
\hline Autoconsciência & $0,59 * * *$ & 0,11 & $-0,39 * * *$ & $0,25^{* *}$ & $0,19 *$ \\
\hline
\end{tabular}

Nota. $* p<0,05 ; * * p<0,005 ; * * * p<0,001$

porém, com fraca magnitude. Realização mostrou-se significativamente associada com mindfulness, atenção e produção de novidades; ao passo que Socialização apresentou correlação com atenção, e Extroversão com produção de novidades. Todas essas últimas com magnitudes fracas de associação.

Do ponto de vista dos fatores da MAP, mindfulness mostrou-se associado com abertura à experiência e realização. Atenção e produção de novidades mostraram associações significativas com quase todos os traços de personalidade, diferenciando-se apenas na magnitude da associação com o fator socialização. Aceitação, por sua vez, mostrou-se significativamente associado com neuroticismo e também com realização.

No que se refere ao BES, o afeto positivo mostrou associação fraca, porém significativa, apenas com o fator mindfulness. $\mathrm{O}$ afeto negativo também resultou em correlação fraca, porém negativa e significativa com o fator aceitação e com atenção plena geral. A atenção plena geral mostrou-se independente do afeto positivo, porém, correlacionou-se fraca e positivamente com satisfação de vida. Satisfação de vida mostrou-se significativamente associada com a atenção plena, apresentando correlações fracas, porém positivas, com mindfulness, atenção e produção de novidade. Apenas aceitação não apresentou associação com satisfação de vida.

A respeito das associações com a atenção, o fator mindfulness foi o que mais apresentou associação com suas diferentes habilidades, com exceção de atenção dividida. A atenção geral da BPA recebeu o maior escore nesse âmbito, apesar da fraca magnitude, seguido da atenção alternada e da concentrada. Houve associação fraca, porém significativa, entre atenção geral e o fator atenção. A atenção dividida apresentou correlação significativa apenas com o fator produção de novidades, ao passo que a atenção plena geral se mostrou significativamente associada com a atenção alternada e geral.

No que diz respeito às associações entre mindfulness einteligência emocional, as correlações encontradas variaram de fraca a moderada. Autoconsciência 
apresentou a correlação de mais forte magnitude com o fator mindfulness, seguida de empatia e automotivação. Aceitação apresentou correlações fracas, porém significativas e negativas tanto com empatia quanto com autoconsciência. Produção de novidades também se mostrou associado à inteligência emocional, apesar da fraca magnitude. Apenas autocontrole não se associou à produção de novidades. $\mathrm{O}$ fator atenção mostrou-se significativamente associado apenas com autocontrole.

\section{Discussão}

Este estudo objetivou encontrar evidências de validade para a medida de atenção plena (MAP), baseando-se na sua associação com outras variáveis, sendo elas: traços de personalidade, bem-estar subjetivo, atenção e inteligência emocional. No que se refere às correlações com os traços de personalidade, os resultados, de forma geral, mostraram-se compatíveis com os reportados na literatura internacional (Baer et al., 2006; Brown \& Ryan, 2003; Giluk, 2009; Latzman \& Masuda, 2013; Siegling \& Petrides, 2014). Isso indica que os dados obtidos com a MAP imprimem padrões também observados em outros estudos envolvendo o construto mindfulness.

O fenômeno mindfulness mostrou-se amplamente correlacionada aos traços de personalidade, entretanto, o padrão da força dessas associações indica que se trata de construtos diferentes, o que é favorável para as hipóteses deste estudo. Quatro fatores gerais destacaram-se no âmbito das associações (neuroticismo, realização, socialização e abertura), conforme era esperado, em observância que esses traços parecem moderar (Vibe et al., 2015), em alguma medida, três importantes aspectos: a) a busca ao estado ou às práticas de mindfulness, b) a adesão (continuidade) ao estado ou às práticas, e ainda c) a percepção dos efeitos de ambos (estado ou práticas). Esse resultado reitera as pontuações de Khazan (2013).

No tocante à moderação, é possível exemplificar que ao se considerar que a prática de mindfulness exige que o praticante dedique algum determinado tempo a ela, pode-se pensar que indivíduos com altos escores no fator abertura sejam mais propensos a buscar e dar continuidade à prática (Van den Hurk et al., 2011) ou ao estado. Essa hipótese segue o argumento de Nunes et al. (2010), que enfatizam a preferência pela convencionalidade nas crenças e atitudes em indivíduos com baixa abertura. Igualmente, vale lembrar que como prática ou estado, aumenta-se, intencionalmente, o contato voluntário com as experiências e, por isso, o nível no fator abertura poderia até ser aumentado se essa prática ocorrer de forma continuada, especialmente nos casos de sujeitos altos em realização, já que indivíduos com essas condições são descritos como mais determinados e persistentes, diferenciando-se dos demais sujeitos nesse domínio (Nunes et al., 2010).

Nessa mesma direção, em relação à adesão à prática ou ao estado mindful, a autodisciplina e motivação ou, conforme discute Shapiro (2009), a intencionalidade, se torna, em alguma medida, um requisito. Entretanto, indivíduos com altos escores em Realização, por exemplo, podem ter a prática ou estado beneficiados pelo traço. Os resultados nesse âmbito indicaram que há uma correspondência entre o nível de mindfulness e a capacidade de organização, persistência, controle e motivação, características típicas de pessoas altas no traço (Nunes et al., 2010). Além disso, visto de outra forma, a prática regular e continuada de autodisciplina poderia até aumentar escores referentes ao fator realização (Vibe et al., 2015).

O fator neuroticismo mostrou-se negativamente relacionado ao construto mindfulness, o que segue o padrão das pesquisas internacionais (Baer et al., 2006; Brown \& Ryan, 2003; Giluk, 2009; Laztman \& Masuda, 2013; Siegling \& Petrides, 2014). Dessa forma, infere-se que indivíduos com altos escores em mindfulness apresentam menos sofrimento, medo de rejeição, e frustração. Diferentemente, indivíduos com altos escores em neuroticismo serão mais suscetíveis a vivenciarem estresse e menos bem-estar e, por conta do traço, poderão ter dificuldades na adaptação às práticas ou ao estado mindful. Apesar disso, não se deve deixar de mencionar que muitos indivíduos com escores elevados em neuroticismo podem ser beneficiados por esse estado.

No que tange ao traço Extroversão, indivíduos com altos escores, em tese, deverão se esforçar mais para manterem-se concentrados e atentos, o que poderia ser um fator potencial para a desistência da prática ou mesmo falta de curiosidade em relação ao estado. Assim, ao considerar a figura de um meditador (alguém que pratica algum tipo de meditação com frequência) como um modelo representativo de altos escores em mindfulness (Baer et al., 2006; Barros et al., 2015; Lau et al., 2006), espera-se que esses indivíduos apresentem baixos escores em extroversão, já que não reatividade às experiências (Baer et al., 2006) e menor impacto de recompensas imediatas (Teper \& Inzlicht, 2013) são atributos de indivíduos com altos escores no construto. 
Essas características estão associadas à extroversão, traço de personalidade que representa a quantidade das interações interpessoais, ao nível da atividade e estimulação (Nunes et al., 2010).

No tocante às associações com afetos, o padrão dos resultados mostrou-se consistente com uma parte das pesquisas internacionais (Baer et al., 2006; Brown \& Ryan, 2003; Giluk, 2009; Jimenez, Niles, \& Park, 2010; Kiken \& Shook, 2014; Vibe et al. 2015) e nacionais (Barros et al., 2014; 2015), apesar da fraca magnitude obtida. Nas pesquisas internacionais, os afetos negativos se associaram mais forte e negativamente com mindfulness do que os positivos, mesma tendência obtida na corrente pesquisa. No entanto, a fraca magnitude da associação entre o fator mindfulness e o afeto positivo mostrou-se diferente do obtido em outros estudos (Brown \& Ryan, 2003; Giluk, 2009; Jimenes, Niles, \& Park, 2010; Kiken \& Shook, 2014), o que indica independência entre essas variáveis.

Esse padrão de independência entre mindfulness e afeto positivo, entretanto, mostrou-se convergente com o estudo de Kiken e Shook (2014), que reportaram correlação fraca entre afeto positivo, a FFMQ e a MAAS. Igualmente, na adaptação brasileira de Barros et al. (2014), a faceta não julgar (FFMQ) apresentou correlação fraca com afeto positivo. Dessa seara pode-se intuir que ter maior consciência das próprias experiências não implica, necessariamente, na percepção ou regulação de mais afetos positivos, já que os afetos apresentam flutuação por conta das vivências cotidianas (Zanon, Dellazana-Zanon, \& Hutz, 2014). Uma hipótese para esse resultado diz respeito às diferenças dos aspectos abarcados nas escalas de mindfulness, as quais avaliam aspectos variados do construto, ao passo que a MAP integra diversos desses elementos. Assim, por entender que essas diferenças facilmente se refletem nos padrões de correlações dos instrumentos, sugere-se que esse resultado seja pesquisado futuramente.

A dimensão cognitiva do bem-estar subjetivo mostrou-se associada à mindfulness, similarmente aos resultados reportados na literatura (Baer et al., 2006; Barros et al., 2014; 2015; Brown \& Ryan, 2003; Giluk, 2009; Kiken \& Shook, 2014; Vibe et al. 2015.). Esse resultado indica que maiores níveis de mindfulness possuem alguma correspondência com uma visão menos distorcida da própria vida. A postura de maior consciência, curiosidade e aceitação das pessoas com altos escores em mindfulness pode contribuir para que as experiências dos diversos domínios da vida possam ser compreendidas de modo abrangente, de forma que a possível visão negativa sobre algum domínio não enviese os demais. Porém, vale mencionar que a satisfação de vida também pode ser explicada pela genética e pelos traços de personalidade (Hutz, Zanon, \& Bardagi, 2014).

No tocante às correlações com atenção, o fator mindfulness foi o que mais se mostrou associado às habilidades de concentrar e alternar, tal como o esperado, apesar da escassa quantidade de pesquisas científicas que tenham testado essa mesma associação, e que poderiam respaldar ainda mais esse achado. Vale lembrar que a magnitude das associações entre o fenômeno mindfulness e as habilidades da atenção, neste estudo, mostraram-se mais elevadas do que as reportadas por Schmertz (2006) Uma possível razão para isso reside no fato de que a MAP avalia o fenômeno mindfulness como um estado, e não uma característica disposicional.

Nesse sentido, em alguns momentos, como por exemplo, ao tomar decisões ou avaliar uma situação, pode ser importante incluir outros aspectos ou pontos de vista, o que exigiria uma mudança da exclusividade dada ao objeto da atenção focada para outros objetos do ambiente ou da consciência, dividindo-se ou alternando-se, voluntariamente, a atenção. Além disso, em alguns momentos, como os de distração, recrutar a consciência para devolver a atenção para o que se está fazendo poderia ser considerado uma forma de regulação da atenção. Assim, diante da percepção de vagueação da atenção, pode-se manejar o seu foco, voltando-o de diferentes estímulos (dividida e alternada), para uma tarefa específica (concentrada), promovendo-se maior sensibilidade a estímulos específicos e facilitando-se o engajamento com a mesma, tal como discutido por Baer et al., (2004).

A respeito da fraca associação entre o fator atenção da MAP e o fator atenção da BPA, pode-se hipotetizar que o primeiro instrumento objetiva captar mais o aspecto da regulação da atenção, ao passo que o segundo avalia outras habilidades típicas da atenção, tais como concentrar, dividir e alternar o seu foco. Isso poderia justificar a magnitude da associação destacada nesse âmbito.

Produção de novidades foi o único fator da atenção plena que apresentou associação significativa com a atenção dividida. Esse resultado mostra-se congruente, uma vez que para produzir novidades no ambiente, faz-se necessário explorá-lo, buscando-se atrair diferentes e novos objetos para a consciência. Igualmente, o fato de a atenção dividida ter sido a habilidade menos associada com a mindfulnes também se mostra conforme 
as expectativas de associação, na medida em que esse é o tipo de habilidade da atenção que poderia sugerir vagueação, indicando que o indivíduo não está engajado com a atividade corrente, tal como preconizado por Baer et al. (2004).

A respeito das correlações com inteligência emocional, os resultados mostraram-se consistentes com as hipóteses e com outros estudos (Baer et al., 2006; Brown \& Ryan, 2003). Autoconsciência foi o fator que apresentou mais forte associação com mindfulness, o que também foi reportado em Brown e Ryan (2003), e em Baer et al., (2006). Esse resultado parece apropriado com a literatura do campo da atenção plena, já que a autoconsciência é um aspecto fundamental da atenção plena (Baer et al., 2004; Brown \& Ryan, 2003). Diferentemente, houve menor associação com o fator sociabilidade, o que mostra consonância com o obtido no fator socialização, do instrumento de personalidade, indicando que a capacidade de socializar mostra-se inferior nos indivíduos com escores mais elevados em mindfulness, o que pode ser validado com o fato de que observar a si próprio, tal como ocorre nas práticas meditativas, por exemplo, costuma ser uma atividade altamente individual. Captar essa essência é fundamental para o reconhecimento da qualidade das dimensões avaliadas pela MAP.

No que se refere à associação negativa entre aceitação e autoconsciência, o resultado foi, de certa forma, inesperado, no entanto, ele indica que ter consciência não implica, a priori, em aceitar aquilo que se torna consciente. Diferentemente, pode-se hipotetizar que quem opta por treinar a aceitação, por exemplo, por meio de algumas práticas meditativas, em alguma medida, concorda que mais aceitação se faz necessária em sua vida de forma geral. Esse resultado também corrobora os achados de Ruiz et al. (2013). Além disso, deve-se considerar que a correlação negativa pode ter ocorrido em resposta ao componente desadaptativo da autoconsciência (Boyraz \& Waits, 2015; DaSilveira, DeSouza, \& Gomes, 2015).

Sobre a associação negativa entre aceitação e empatia, Siqueira et al., (1999) descreveram que empatia se refere à facilidade de identificar os sentimentos, desejos, motivações e emoções dos outros. Considerando que mindfulness diz respeito a voltar-se a consciência para as próprias experiências, justifica-se, dessa maneira, tal resultado negativo. Igualmente, esse resultado parece alinhado com o que ocorreu na pesquisa de Boyraz e Waits (2015), no qual a autoaceitação mostrou-se inversamente relacionado com empatia.
Para concluir, em observância aos resultados obtidos nas correlações, pode-se dizer que a MAP possui evidência de validade baseada na associação com medidas de outras variáveis, sendo convergente com a personalidade e inteligência emocional, além de divergente com o bem-estar subjetivo e com atenção. Esses resultados são indicativos de que a MAP é um instrumento adequado para avaliar o construto que ela se propõe.

\section{Considerações Finais}

As hipóteses de associação entre mindfulness e traços de personalidade, afetos, satisfação de vida, atenção e inteligência emocional, foram amplamente corroboradas neste estudo. Além disso, as correlações obtidas seguiram os padrões reportados na literatura científica internacional e nacional, de forma que tais associações foram bastante congruentes e interpretáveis. Abertura e Realização mostraram-se como os traços de personalidade mais associados à mindfulness, sendo variáveis que, se forem consideradas por praticantes, profissionais da saúde e instrutores de mindfulness, poderão contribuir com a busca e adesão às práticas ou ao estado mindful. Vale destacar que a amostra participante é uma limitação deste estudo, já que foi composta, principalmente, por indivíduos com alta escolaridade, característica que pode ter enviesado os resultados obtidos correntemente. Além disso, sugere-se que estudos futuros busquem verificar a capacidade preditiva dos traços de personalidade em relação aos componentes medidos pela MAP.

\section{Referências}

Baars, B., J. (1997). Some Essential Differences between Consciousness and Attention, Perception, and Working Memory. Consciousness and Cognition 6, 363371. Recuperado de http://selfpace.uconn.edu/ class/ccs/Baars1997AttentionAwarenessCcs.pdf

Baer, R. A., Smith, G. T., \& Allen, K. B. (2004). Assessment of mindfulness by self-report: The kentucky inventory of mindfulness skills. Assessment, 11(3), 191-206. doi: 10.1177/1073191104268029

Baer, R. A., Smith, G. T., Hopkins, J., Krietemeyer, J., \& Toney, L. (2006). Using self-report assessment methods to explore facets of mindfulness. Assessment, 13(1), 27-45. doi: 10.1177/1073191105283504 
Barros, V. V., Kozasa, E. H., Souza, I. C. W., \& Ronzani, T. M. (2014). Validity evidence of the brazilian version of the five facet mindfulness questionnaire (FFMQ). Psicologia: Teoria e Pesquisa, 30(3), 317-327. doi: 10.1590/S0102-37722014000300009

Barros, V. V., Kozasa, E. H., Souza, I. C. W., \& Ronzani, M. T. (2015). Evidences of validity of the brazilian version of the Mindful Attention Awareness Scale (MAAS). Psicologia: Reflexão e Crítica, 28(1), 87-95. doi: 10.1590/1678-7153.201528110

Bergomi, C. Tschacher, W., \& Kupper, Z. (2012). Assessment of mindfulness with self- report measures: Existing scales and Open issues. Mindfulness, 1-12. doi: 10.1007/s12671-012-0110-9

Bishop, S. R., Lau, M. A., Shapiro, S. L., Carlson, L., Anderson, N. D., Carmody, J., \& Devins, G. (2004). Mindfulness: A proposed operational definition. Clinical Psychology, 11(3), 230-241. doi:10.1093/ clipsy.bph077

Boyraz, G., \& Waits, J. B. (2015). Reciprocal associations among self-focused attention, self-acceptance, and empathy: A two-wave panel study. Personality and Individual Differences. 74, 84-89. doi: 10.1016/j. paid.2014.09.042

Brown, K. W., \& Ryan, R. M. (2003). The benefits of being present: Mindfulness and its role in psychological well-being. Journal of Personality and Social Psychology, 84(4), 822-48. doi: 10.1037/0022-3514.84.4.822

Diener, E., Emmons, R. A., Larsen, R. J., \& Griffin, S. (1985). The satisfaction with life scale. Journal of Personality Assessment, 49, 71. Recuperado de http:// internal.psychology.illinois.edu/ ediener/Documents/Diener-Emmons-Larsen-Griffin_1985.pdf

Diener, E., Lucas, R. E., \& Oishi, S. (2005). Subjective well-being: The science of happiness and life satisfaction. Em C. R. Snyder \& S. J. Lopez (Eds.). Handbook of positive psychology (2 Ed.), (pp. 63-73). New York, NY: Oxford University Press.

Giluk T. L. (2009). Mindfulness, Big Five personality, and Affect: A meta-analysis. Personality and Individual Differences; 47(8), 805-811. doi: 10.1016/j. paid.2009.06.026

Hutz, C. S., Zanon, C., \& Bardagi, M. P. (2014). Satisfação de vida. Em Hutz, Claudio. S. (Eds.). Avaliação em Psicologia Positiva. Porto Alegre: Artmed. 121-146.
International Test Commission (2014). ITC Statement On the Use of Tests and Other Assessment Instruments for Research Purposes. Recuperado de https://www.intestcom.org/files/statement_ using_tests_for_research.pdf

Jimenez, S. S., Niles, B. L., \& Park, C. L. (2010). A mindfulness model of affect regulation and depressive symptoms: Positive emotions, mood regulation expectancies, and self-acceptance as regulatory mechanisms. Personality and Individual Differences, 49(6), 645-650. doi: 10.1016/j.paid.2010.05.041

John, O. P., Naumann, L. P., \& Soto, C. J. (2008). Paradigm shift to the integrative Big Five trait taxonomy. Em Handbook of personality: Theory and research (pp. 114-158). Guilford Press New York, NY. Recuperado de http://www.ocf.berkeley. edu/ johnlab/2008chapter.pdf

Kabat-Zinn, J. (2003). Mindfulness-based interventions in context: Past, present, and future. Clinical Psychology: Science and Practice, 10(2), 144-156. doi: 10.1093/ clipsy/bpg016

Khazan, I., Z. (2013). The clinical handbook of Biofeedback-A step-by-step guide for training and practice with Mindfulness. Willey-Blackwell. Chichester, UK

Kiken L. G., \& Shook, N. J. (2014). Does mindfulness attenuate thoughts enphasizing negativity, but not positivity? Journal ofResearch in Personality, 53, 22-30. doi: 10.1016/j.jrp.2014.08.002

Langer, E. J. (2014) Mindfulness forward and back. Em Ie, A., Christelle, T., N. \& Langer, E., J. (Eds.), Wiley Blackwell Handbook of Mindfulness (pp. 7- 20) Chichester, UK: John Wiley \& Sons, Ltd. doi: 10.1002/9781118294895.ch1.

Latzman, R., D., \& Masuda, A. (2013). Examining mindfulness and psychological inflexibility within the framework of Big Five personality. Personality and Individual Differences, Volume 55, Issue 2, July 2013, 129-134. doi: 10.1016/j.paid.2013.02.019

Lau, M. A., Bishop, S. R., Segal, Z. V., Buis, T., Anderson, N. D., Carlson, L., \& Carmody, J. (2006). The Toronto Mindfulness scale: Development and validation. Journal of Clinical Psychology, 62(12), 1445-1467. doi: 10.1002/jclp.20326/pdf

Leahy, R. L., Tirch, D., \& Napolitano, L. A. (2013). Regulação Emocional em Psicoterapia: um guia para o 
terapeuta cognitivo- comportamental. Trad. Ivo Oliveira. Porto Alegre: Artmed.

Mrazek, M. D., Smallwood, J., \& Schooler, J. W. (2012). Mindfulness and mind-wandering: Finding convergence through opposing constructs. Emotion, 12, 442-448. doi: 10.1037/a0026678

Nunes, C. H. S. S., Hutz, C. S., \& Nunes, M. F. O. (2010). Bateria Fatorial de Personalidade (BFP): Manual técnico. São Paulo: Casa do Psicólogo.

Pires, J. G. (2016). Construção e busca de evidências de validade de uma medida de atenção plena para adultos (Dissertação de mestrado). Programa de Pós-graduação em Psicologia. Universidade Federal de Santa Catarina. Florianópolis, SC. Brasil. Recuperado de http:// tede.ufsc.br/teses/PPSI0699-D.pdf

Rueda, F. J. M. (2013). Bateria Psicológica para Avaliação da Atenção - BPA. Manual técnico. São Paulo: Vetor.

Rueda, F. J. M., \& Monteiro, R., M. (2013). Bateria Psicológica para Avaliação da Atenção (BPA): desempenho de diferentes faixas etárias. Psico-USF, v. 18, n. 1, p. 99-108. Recuperado de http://www. scielo.br/pdf/pusf/v18n1/v18n1a11.pdf

Ruiz, F. J., H, A. I. L., Luciano, C., Cangas, A. J., \& Beltrán, I. (2013). Measuring experiential avoidance and psychological inflexibility: The Spanish version of the Acceptance and Action Questionnaire-II. Psicothema, 25(1), 123-129. doi: 10.7334/ psicothema2011.239

Salovey, P., \& Mayer, J. D. (1995). Emotinalintellience and the construction and regulation of feelings. Applied and Preventive Psychology, 4, 198-208. Recuperado de http://www.unh.edu/emotional_intelligence/EIAssets/EmotionalIntelligenceProper/EI1990\%20 Emotional\%20Intelligence.pdf

Shapiro, S. L. (2009). The integration of mindfulness and psychology. Journal of Clin. Psychol., 65, 555560. doi: 10.1002/jclp.20602

Siegling, A. B., \& Petrides, K. V. (2014) Measures of trait mindfulness: Convergent validity, shared dimensionality, and linkages to the five-factor model. Front. Psychol., 5, 1164. doi: 10.3389/ fpsyg.2014.01164

DaSilveira A., DeSouza M. L., \& Gomes W. B. (2015). Self-consciousness concept and assessment in selfreport measures. Front. Psychol. 6, 930. doi: 10.3389/ fpsyg.2015.00930
Schmertz, S. K. (2006). The Relation between Self-Report Mindfulness and Performance on Tasks of Attention. Thesis, Georgia State University. Recuperado de http://scholarworks.gsu.edu/ psych_theses/30

Silveira A., Castro, T. G., \& Gomes W. B. (2012). Adaptação e validação da Escala Filadélfia de Mindfulness para adultos brasileiros. Psico-USF, 17(2), 215-223. doi: 10.1590/S1413-82712012000200005

Siqueira, M., M., M., Barbosa, N., C., \& Alves, M., T. (1999). Construção e Validação Fatorial de uma Medida de Inteligência Emocional. Psicologia: Teoria e Pesquisa, Vol. 15 n. 2, pp. 143-152. Recuperado de http://www.scielo.br/pdf/ptp/v15n2/a07v15n2. pdf

Sternberg, R. J. (2008). Atenção e Consciência. Em Sternberg, R. J. (Eds.). Psicologia Cognitiva. Porto Alegre: Artmed. pp. 71-114.

Teper, R., \& Inzlicht, M. (2013). Mindful Acceptance Dampens Neuroaffective Reactions to External and Rewarding Performance Feedback. Emotion. 14(1), 105-14. doi: 10.1037/a0034296

Van den Hurk, P. A. M., Wingens, T.,Giommi, F., Barendregt, H. P., Speckens, A. E. M., \& Van Schie, H. T. (2011). On the Relationship Between the Practice of Mindfulness Meditation and Personality - an Exploratory Analysis of the Mediating Role of Mindfulness Skills. Mindfulness (NY) 2(3), 194200. doi: 10.1007/s12671-011-0060-7

Vibe, M., Solhaug, I., Tyssen, R., Friborg, O., Rosenvinge, J. H., Sørlie, T., \& Bjørndal, A. (2015). Does Personality Moderate the Effects of Mindfulness Training for Medical and Psychology Students? Mindfulness, 6(2), 281-289. doi: 10.1007/ s12671-013-0258-y

Watson, D., Clark, L. A., \& Tellegen, A. (1988). Development and Validation of Brief Measures of Positive and Negative Affect: The PANAS Scales. Journal of Personality and Social Psychology. vol. 54. $\mathrm{n}^{\circ}$. 6, 1063-1070. Recuperado de http://www.cnbc. pt/jpmatos/28.Watson.pdf

Zanon, C., Dellazzanna-Zanon, L. L., \& Hutz, C. S. (2014). Afetos positivos e negativos: Definições, avaliações e suas implicações para intervenções. Em Hutz, Claudio. S. (Eds.). Avaliação em Psicologia Positiva. Porto Alegre: Artmed. pp 49-61. 
Zanon, C., \& Hutz, C. S. (2014). Escala de Afetos Posi-

Recebido em: 20/02/2017 tivos e Negativos (PANAS). Em Hutz, Claudio. S. Reformulado em: 19/09/2017 (Eds.). Avaliação em Psicologia Positiva. Porto Alegre: Aprovado em: 06/12/2017 Artmed. pp 63-67.

Sobre os autores:

Jeferson Gervasio Pires é psicólogo, mestre e doutorando em Psicologia pelo Programa de Pós-graduação em Psicologia da Universidade Federal de Santa Catarina (UFSC).

E-mail: jefersongp@gmail.com

ORCID: https://orcid.org/0000-0001-8121-8922

Carlos Henrique Sancineto da Silva Nunes é psicólogo, mestre, doutor em Psicologia pela Universidade Federal do Rio Grande do Sul e professor associado da Universidade Federal de Santa Catarina.

E-mail: carloshnunes@mac.com

ORCID: https://orcid.org/0000-0002-7769-6937

Maiana Farias Oliveira Nunes é psicóloga, mestre, doutora em Psicologia pela Universidade São Francisco e professora ajunta da Universidade Federal de Santa Catarina.

E-mail:maiananunes@mac.com

ORCID: https://orcid.org/0000-0003-4891-5982

Marcelo Marcos Piva Demarzo é médico, doutor em Patologia pela Universidade de São Paulo e docente da Escola Paulista de Medicina da Universidade Federal de São Paulo.

E-mail:marcelodemarzo@gmail.com

ORCID: https://orcid.org/0000-0002-7447-1839

Maria Luiza Bianchi é acadêmica do curso de Psicologia da Universidade Federal de Santa Catarina.

E-mail:marialuiza_b@yahoo.com.br

ORCID: https://orcid.org/0000-0001-6464-6861

Mônica Monteiro Kotzias é acadêmica do curso de Psicologia da Universidade Federal de Santa Catarina.

E-mail:monicakotzias@gmail.com

ORCID: https://orcid.org/0000-0002-3044-2446

Giovania Mitie Maesima Cunha é acadêmica do curso de Psicologia da Universidade Federal de Santa Catarina. E-mail: giovaniamitie@gmail.com

ORCID: https://orcid.org/0000-0003-4347-3601

Contato com os autores:

Campus Reitor João David Ferreira Lima - Trindade. Prédio novo CFH, bloco E, $3^{\circ}$ andar Cx. Postal 476

Florianópolis-SC, Brasil

CEP: 88040-900 